\title{
Análisis del sistema regional de ciencia, tecnología e innovación del Valle del Cauca
}

\author{
HENRY CAICEDO ASPRILLA, MSc., Ph.D. (c) ${ }^{1,2}$ \\ Profesor de Comercio Internacional y Economía del Cambio Tecnológico, \\ Universidad del Valle, Colombia. \\ henry.caicedo@correounivalle.edu.co
}

\section{RESUMEN}

Este trabajo tiene como propósito aplicar la metodología de redes sociales para evaluar si en el Valle del Cauca, se está consolidando un sistema de innovación. Se encontró que en la región hay muy pocas organizaciones que puedan dar soporte en la innovación a las empresas, lo que ha dificultado la consolidación de un sistema fuerte, articulado y que, a diferencia del esquema actual, busque que las empresas se relacionen para brindar servicios empresariales con alto contenido tecnológico que les permita innovar. El estudio concluye que un sistema de innovación en el Valle del Cauca constituye más una aspiración de política que una realidad del desarrollo tecnológico de la región.

Palabras clave. Desarrollo regional; sistemas de innovación; transferencia de tecnología; análisis de redes sociales.

Recibido: 30-jun-11, corregido: 26-abr-12 y aceptado: 26 -jun-12

$$
\text { Clasificación JEL: R58; O31; D85 }
$$

@

\footnotetext{
1 Este documento fue seleccionado en la convocatoria para enviar artículos, Call for Papers, realizada en el marco del "II Simposio Iberoamericano de Estudios Gerenciales: Una mirada interdisciplinar a la innovación", organizado por la revista académica Estudios Gerenciales bajo la dirección de la Facultad de Ciencias Administrativas y Económicas de la Universidad Icesi; el evento tuvo lugar los días 12, 13 y 14 de octubre de 2011, en la ciudad de Cali (Colombia). Este documento fue presentado en las sesiones simultáneas del área de "Modelos de innovación aplicados".

2 Dirigir correspondencia a: Universidad del Valle, Calle 4 B \# 36-oo, Edificio 124, Despacho 1028, Cali, Colombia.
} 


\section{An analysis of the regional system of science, technology, and innovation in the State of Valle del Cauca}

This research work is aimed at applying the social network methodology in order to determine whether an innovation system is increasingly growing stronger in the Valle del Cauca. We find that there are only a few organizations in the region that are capable of supporting business innovation. This has hampered establishing a strong articulated system which, unlike the current environment, is aimed at getting companies to establish links with each other to provide business services with a high technological content that enables them to innovate. We conclude that the idea of innovation systems in the State of Valle del Cauca is more of an aspiration than a reality of technological development in the region.

Keywords. Regional development; innovation systems; technology transfer; social network.

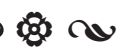

\section{Análise do sistema regional de ciência, tecnologia e inovação do Vale de Cauca}

Este trabalho tem como objetivo aplicar a metodologia das redes sociais para avaliar se, no Vale de Cauca, está se consolidando um sistema de inovação. Descobrimos que na região existem muito poucas organizações que possam dar apoio à inovação nas empresas, o que tem dificultado a consolidação de um sistema forte, articulado e que, ao contrário do esquema atual, procure que as empresas se relacionem para oferecer serviços empresariais com elevado conteúdo tecnológico que lhes permitam inovar. O estudo conclui que um sistema de inovação no Vale de Cauca é mais uma aspiração política do que uma realidade do desenvolvimento tecnológico da região.

Palavras-chave. Desenvolvimento regional; sistemas de inovação; transferência de tecnologia; análise de redes sociais. 


\section{Introducción}

En el año 2000 el gobierno colombiano propuso el documento CONPES 3080 (Consejo Nacional de Política Económica y SocialCONPES, 2000) en el cual se diseñaron los lineamientos de política para conformar los Comités Departamentales de Ciencia y Tecnología. El objetivo de la confirmación de estos comités, era el de constituir y/o consolidar una institucionalidad que estimulara en la sociedad y en la economía de las regiones, el uso y producción del conocimiento a través de los llamados Sistemas Regionales de Ciencia, Tecnología e Innovación (en adelante SRCTI). Este concepto es equivalente al de Sistemas Regionales de Innovación, SRI, propuesto por Cooke, Gómez \& Etxebarria (1997).

En respuesta a los lineamientos de política nacional, en el Valle del Cauca se estimuló el interés por consolidar un sistema de innovación; esta iniciativa en la región parte de reconocer la existencia de organizaciones del conocimiento tales como centros de desarrollo productivo, centros científicos y tecnológicos, universidades, organizaciones promotoras del comercio exterior, así como las que apoyan la gestión empresarial, las que financian la producción, además de un sector productivo que se disputa con Antioquia el segundo lugar en industrialización del país.

Además, en la región, con el fin de fortalecer el SRCTI, se emprendieron múltiples procesos de concertación en el marco del documento Colombia 2019: la formulación del Plan Maestro, la construcción de la Agenda Regional de Ciencia y Tecnología, el desarrollo de la Agenda Interna de Competitividad y Productividad, la elaboración del Plan Bioregión. En todos estos trabajos está presente la idea de lograr hacer del Valle una región próspera basada en el conocimiento.
Desde que el concepto de SRCTI surgió en la agenda de los investigadores de la economía de la innovación, este ha sido tema de investigación, preguntándose si éste es un concepto aplicable en todos los países y regiones, especialmente buscando conocer si en efecto los sistemas de innovación alcanzan un grado de fortaleza y consolidación como para ayudar al desarrollo de las regiones donde toman lugar (Bell \& Pavitt, 1993; Casas, 2001; Cassiolato, 2002; Carlsson, 2006; Edquist, 2001; Freeman, 1987; Yoguel, Borello \& Erbes, 2005).

El propósito del presente trabajo es aplicar la metodología de redes sociales para analizar y describir el sRCTI del Valle del Cauca, con el fin de evaluar si en la región se está consolidando un sistema de innovación con la capacidad de generar y transferir el conocimiento y la tecnología que sirva de soporte o atmósfera industrial a las empresas locales y extranjeras y, por ende, contribuya al desarrollo regional.

Entre los objetivos del trabajo se tienen el identificar las organizaciones y los acuerdos que se establecen entre éstas para generar y/o transferir conocimiento y tecnología en el SRCTI de la región; el determinar el grado de articulación existente entre las organizaciones que apoyan el proceso de innovación y gestión del conocimiento en el departamento; y describir la estructura del SRCTI del Valle en términos del poder y jerarquía de las organizaciones.

El trabajo se compone de cinco partes, siendo la primera esta introducción. En la segunda se especifica la relación entre los SRCTI y las redes. En la tercera se describe la metodología de trabajo. En la cuarta se analizan los resultados, y por último, en la quinta se plantean un conjunto de conclusiones y posibles líneas de investigación. 
1 Los sistemas regionales de ciencia, tecnología e innovación

Un sistema de innovación es el conjunto de instituciones que, en conjunto e individualmente, contribuyen al desarrollo y difusión de nuevas tecnologías. Estas instituciones proporcionan el marco dentro del cual los gobiernos forman e implementan políticas para influenciar los procesos de innovación. Como tal, es un sistema de instituciones interconectadas para crear, almacenar y transferir el conocimiento, las habilidades y las herramientas para definir nuevas tecnologías (Metcalfe, 1998). Cuando esta institucionalidad se localiza en las regiones, lo que se pretende es resaltar que la proximidad entre los agentes permite una mayor interacción y difusión del conocimiento tácito, que por definición, al no estar codificado, requiere de una relación cara a cara para incorporarlo a la innovación (Cooke, 2004; DeBresson \& Amesse, 1991; Gertler \& Levitte 2003; Iammarino, 2005; Morgan, 2004).

El énfasis de la dinámica y funcionamiento de los SRCTI está en el proceso de aprendizaje (Lundvall, 1992); el cual es el soporte de la creación de conocimiento, a la vez que de la transferencia de tecnología del exterior y de la difusión de las innovaciones (Lundvall \& Lorenz, 2009). El aprendizaje permite que estos procesos ocurran a un costo menor, debido a la proximidad de los agentes (Boschma, 2005), por la mayor posibilidad de absorber las externalidades de la tecnología (Jaffe, Trajtenberg \& Henderson, 1993), y también por la posibilidad de combinar el aprendizaje derivado del uso del conocimiento explícito o basado en la ciencia, y el conocimiento tácito o basado en la experiencia (Lundvall \& Lorenz, 2009).

Lundvall (2007), en el afán de definir y precisar el marco analítico de los sistemas de innovación, intenta trazar la frontera con respecto a los demás sistemas de la economía y sociedad. Para ello, identifica que el tejido empresarial y las relaciones que se establecen con las organizaciones científicas y tecnológicas se constituyen en el core de un SRCTI. Allí donde se pueden establecer los vínculos que el sistema de innovación crea con las demás esferas de la sociedad, como la del mercado de trabajo, el medio ambiente, el sistema financiero, etc.

Los sistemas de innovación cumplen varios papeles o roles. Aquí interesan dos perspectivas: la institucional y la operacional. Desde la lógica institucional, un SRCTI contribuye a reducir la incertidumbre, proporcionar información, facilitar la cooperación y reducir los conflictos y brindar incentivos para la innovación (Edquist \& Johnson, 1997). Desde la lógica operacional, el SRCTI contribuye a facilitar las interacciones entre agentes, proporcionar recursos para la innovación y dinamizar la transferencia de tecnología y conocimiento desde y hacia el exterior de la región (Hekkert, Suurs, Negro, Smits \& Kuhlmann, 2007).

El cumplimiento de las funciones en un SRCTI depende de las proximidades geográfica, social, institucional, organizacional y cognitiva de los agentes (Boschma, 2005). En cuanto a la localización geográfica, demanda que los agentes estén ubicados en un radio de distancia que permita que en un día se puedan efectuar viajes en automóvil de ida y regreso (Andersson \& Karlsson, 2004). Así mismo, la proximidad social implica que los agentes compartan un capital social común (Lundvall, 2002), a la vez que la cercanía organizacional impone que en la región se disponga de las organizaciones necesarias para acometer con probabilidad de éxito el proceso de innovación (Velasco, 2002). De igual forma, en relación a la proximidad institucional, los agentes deben compartir normas, valores y leyes (Lundvall, 2007) y, 
finalmente, la cercanía cognitiva es la que permite el flujo e intercambio de conocimiento entre los agentes de un territorio (Boschma, 2005; Lorentzen, 2009).

Un sistema de innovación será débil o frágil cuando la proximidad sea baja o mínima (Lorentzen, 2009), o cuando no pueda desempeñar las funciones anteriormente a descritas. Esta dificultad surge cuando se identifican las fallas de los sistemas de innovación, como por ejemplo, cuando no existen las organizaciones necesarias o, cuando existen, pero no son adecuadas para emprender la innovación. De igual forma, existen fallas cuando las interacciones o vínculos entre los actores no conducen a la transferencia de conocimiento; y por último, el SRCTI falla cuando las instituciones no estimulan la generación de conocimiento y tecnología (Lundvall, 2007; Velasco, 2002).

\subsection{Los SRCTI como redes de conocimiento e innovación}

Para que un SRCTI alcance un nivel de madurez, deberá satisfacer dos condiciones: en primer lugar, que existan las organizaciones e instituciones necesarias, y en segundo lugar, que entre los agentes se configuren procesos de articulación o redes que garanticen que haya una circulación del conocimiento desde las organizaciones que lo producen hasta aquellas que lo transforman en bienes, servicios e información (Cooke, 2004; Isaksen 2001; Lundvall, 2007). Del cumplimiento de estas dos condiciones se desprende que un SRCTI bien consolidado es aquel que se comporta como una red (Casas, 2001; Koschatzky, 2002; Navarro \& Gibaja, 2009).

\subsection{Los actores y los vínculos en una red de un sistema de innovación}

Existe un relativo acuerdo en que los componentes de un SRCTI son cinco: el entorno institucional, el científico y tecnológico, el productivo, el financiero y, por último, el de intermediación (Asheim \& Isaksen, 2001; Edquist \& Johnson, 1997; Fernández \& Castro, 1995; Lundvall, 1992; Velasco, 2002).

Igualmente, hacen parte del entorno institucional en un SRCTI, las organizaciones del Estado central, regional o local, que tienen como función diseñar y aplicar las leyes, planes y programas que estimulen la innovación (Buesa, Martínez, Heijs \& Baumert, 2002; Velasco, 2002). Por su parte, los actores que constituyen el entorno científico y tecnológico son las organizaciones encargadas de la generación de innovaciones y producción del conocimiento (Fernández \& Castro, 1995); entran en esta categoría las universidades, los laboratorios de investigación adscritos a una agencia gubernamental o empresa, los centros tecnológicos y los centros productivos (Sánchez \& Elena, 2006). Constituyen el entorno empresarial o productivo las organizaciones que cumplen el papel de materializar el conocimiento en productos, procesos y servicios; naturalmente, este grupo lo constituyen las empresas, los gremios y los sindicatos (Fernández \& Castro, 1995; Lundvall, 2007).

En el entorno de intermediación se localizan las organizaciones como agencias de innovación, los consultores y los proveedores de servicios empresariales (Fernández \& Castro, 1995; Velasco, 2002). Por último, hacen parte del entorno financiero las instituciones proveedoras de capital de riesgo o semilla, aquellas que acompañan al empresario en el riesgo que implica un proceso de innovación (Buesa et al., 2002; Velasco, 2002). Como lo planteara Schumpeter (1996), los banqueros son los porteros del desarrollo en cuanto que son los que pueden frenar o no el emprendimiento y desarrollo de innovaciones.

En un SRCTi las organizaciones se articulan en una red cuyos objetivos fundamen- 
tales son la circulación del conocimiento y la generación de innovaciones (Gertler \& Levitte, 2003). En el análisis de redes importan tanto las características de agentes y organizaciones como los vínculos que se establecen (DeBresson \& Amesse, 1991; Freeman, 1991; Powell, 1990). Con base en esta propuesta, Koschatzky (2002) ha identificado un conjunto de modalidades de transferencia de tecnología que estimula la formación de redes: Joint Ventures para emprender proyectos de investigación, acuerdos multilaterales en $\mathrm{I}+\mathrm{D}$ entre la universidad y el Estado, acuerdos para el intercambio de tecnologías entre empresas, convenios para realizar inversiones directas inducidas por la tecnología, acuerdos de licencia y second sourcing, procesos de subcontratación, redes de proveedores, colectivos de investigación impulsados por la administración pública, infraestructura basada en las TICs, el intercambio tecnológico y científico orientado hacia la cadena de valor, acuerdos informales de transferencia de conocimiento. Llegados a este punto, se plantea la primera hipótesis del trabajo:

Hipótesis 1 (H1). El sRCTI del Valle del Cauca es un sistema de innovación frágil y débil debido a que, si bien en la región se identifican organizaciones e instituciones en cada uno de los entornos que componen un SRCTI, los acuerdos y vínculos que se generan entre éstas no tienen como principal objetivo la producción y/o la transferencia de tecnología, más bien se firman y establecen para la transferencia de servicios empresariales que, a lo sumo, contribuyen al mejoramiento continuo de las empresas más que a la innovación.

\subsection{La estructura de red de los SRCTI}

Pero si bien la presencia de las organizaciones es una condición necesaria para la consolidación de un sistema de innovación, no es suficiente. Como se sabe, la condición más importante de un SRCTI es la de poseer una configuración de redes que tenga como objetivos la innovación y la generación de conocimiento (Casas, 2001; Lorentzen, 2009). Los agentes se articulan en forma de red debido a que el conocimiento y la innovación son procesos que no responde a la dinámica de la competencia pura porque no se pueden apropiar toda la rentabilidad esperada de la inversión en investigación y desarrollo (Dosi, 1999; Lundvall, 1999; Romer 1986; Utterback, 2001).

En un SRCTI se distinguen dos clases de redes bien diferenciadas: las redes interempresariales, que se caracterizan por una relación estrecha entre productores y usuarios, la presencia de efectos de aprendizaje, la obtención de ventajas de especialización y coordinación, así como por una subcontratación cooperativa a largo plazo (Asheim \& Coenen, 2004). Esta clase de redes son las llamadas clúster por Porter (1997) o distritos industriales por Becattini (1990).

Los distritos industriales son la red de instituciones que sirve de soporte a la innovación y producción del conocimiento. Esta se caracteriza por generar y crear la atmósfera para la innovación, servir de vehículo para la circulación del conocimiento, crear el capital social para aumentar las ganancias de la innovación, disminuir el riesgo que entraña la producción de bienes con un alto componente tecnológico, facilitar la transferencia y adaptación de la tecnología a las empresas, fundamentalmente las más pequeñas, y disminuir los costos de transacción en las negociaciones de bienes y factores tecnológicos (Asheim \& Isaksen, 2002; Cooke, 2001; Lundvall, 2007; Lundvall \& Johnson, 1994; Morgan, 1997). En esta perspectiva, para que un SRCTI se comporte como una red de conocimiento debe cumplir las siguientes características: 
- Estar altamente conectado. Esto significa que en un SRCTI, los agentes deberán estar articulados a través de procesos de colaboración para la transferencia y o producción de conocimiento e innovaciones (Andersson \& Karlsson, 2004);

- Para que un sistema se fortalezca las relaciones entre los agentes deben ser recurrentes, redundantes y recíprocas, porque así se garantiza el aprendizaje derivado de las relaciones cara a cara, basadas en el aprovechamiento del conocimiento tácito de los agentes (Lundvall, 2002, 2007; Lundvall \& Lorenz, 2009);

- En un SRCTI, entre mayor sea el número de relaciones, mayor será la posibilidad de comunicación y menor será la distancia o pasos que tenga que llevar a cabo un agente para establecer vínculos con otro; todo esto llevará a que el sistema sea más fuerte, denso, con mayor nivel de alcance y fluidez de información y conocimiento entre los agentes, a la vez que será menos fragmentado (Cantner, Meder \& Ter Wal, 2010).

Con estas condiciones, el sistema podrá permitir más procesos de producción, circulación y explotación del conocimiento y la tecnología (Florida, 1995; Gertler \& Levitte, 2003; Lundvall, 2007; Morgan, 1997). Dado lo anterior, se plantea la segunda hipótesis de esta investigación:

Hipótesis 2 (H2). El SRCTI del Valle del Cauca es un sistema de innovación débil porque presenta una baja conectividad y cohesión debido a que existe un bajo nivel de vinculación y relacionamiento entre las organizaciones; así mismo, el sistema es frágil porque es fragmentado, lo cual implica que las organizaciones tienen que hacer grandes esfuerzos para establecer relaciones con otras, lo que necesariamente se convierte en un freno u obstáculo para que el sRCTI cumpla las funciones de apoyar al desarrollo regional.
Una segunda característica importante de un SRCTI es la identificación de la estructura. En este aspecto importa entender el papel que juega cada organización y el poder que esta tiene dentro del sistema (Cooke et al., 1997). Un SRCTi será más fuerte entre menores sean los costos de transacción, menor propensión haya para el oportunismo, mayor sea la cooperación y la confianza, y mayor sea la oferta o disponibilidad de organizaciones proveedoras de tecnologías (Asheim \& Isaksen, 2001; Lundvall, 2007).

Desde la lógica de la estructura, estas condiciones implican que un sistema será más fuerte entre menor sea la jerarquía o poder de las organizaciones y menor sea la especificidad de los activos, o lo que es lo mismo, menor sea la relación de dependencia entre las organizaciones en un proceso de colaboración, de generación y/o transferencia de tecnología (Lundvall, 2002; Velasco, 2002). En este marco de análisis, se propone la tercera hipótesis del presente trabajo:

Hipótesis 3 (H3). En el Valle del Cauca el SRCTI es frágil porque existen organizaciones con poder significativo, de tal forma que si se eliminaran, fragmentarían el sistema como un todo; de igual manera, el poder concentrado en pocas organizaciones, hace que el flujo de información y conocimiento sea vulnerable a la presencia de costos de transacción, a asimetrías de información y a procesos de cooperación ineficientes.

\section{Metodología}

Con el fin de verificar las hipótesis y alcanzar los objetivos, en el presente trabajo se aplicó el análisis de redes sociales proveniente de la sociología estadística y matemática (Casti, 1995; Hanneman, 2000). Se eligió esta metodología porque permite identificar los actores y clasificarlos por funciones, a la vez que valora los actores más por la capacidad que 
tienen para relacionarse con otros, que por los atributos (Granovetter, 1985; Hanneman, 2000). El análisis de redes sociales permite identificar la existencia y las principales características de la red, en este sentido, se podrá mostrar si la red es de innovación y conocimiento. A continuación se describe la aplicación de la metodología.

\subsection{La identificación y selección de los ac- tores}

En un SRCTI se distinguen dos grupos de actores, las redes de empresas y las de las infraestructuras de innovación (Lundvall, 2007). En este trabajo se tomaron como organizaciones del SRCTi del Valle del Cauca sólo las del segundo grupo, es decir, aquellas que tuvieran en su misión, visión y objetivos, la generación, producción, circulación y transferencia hacia las empresas del conocimiento, ya sea el generado en la región o el producido fuera de ella. También se incluyeron las organizaciones del tipo de gremios y sindicatos, en el entendido que estas contribuyen a la acumulación de capital y al desempeño de las empresas.

La metodología de redes no admite, de plano, selección de muestras (Molina, 200o), porque una red se construye a partir de la referencia que haga un actor que tenga o haya tenido relación con otro (Hanneman, 200o). En el presente trabajo se siguió como estrategia de elección de organizaciones y la selección por referencia.

Como resultado de la aplicación de la lista de referidos, se seleccionaron 102 organizaciones, de las cuales respondieron 78 , lo que constituye una tasa de respuesta del $77 \%$. Estas categorizaciones se hicieron siguiendo dos atributos: en primer lugar, que pudieran clasificarse en uno de los entornos que componen un SRCTI; en segundo lugar, que se pudiera identificar en la misión y objetivos de la organización, su relación con los procesos de innovación y apoyo al tejido empresarial.

Una vez identificadas las organizaciones se procedió a hacer lo propio con los vínculos. Se eligieron como relaciones relevantes aquellas que cumplieran con al menos dos de los siguientes requisitos: primero que los acuerdos debían estar permitidos por la Ley 29 (Congreso de la Republica de Colombia, 1990) y sus decretos reglamentarios, y segundo que las relaciones entre las organizaciones debían estar orientadas a facilitar la transferencia, la producción. En suma, la gestión de la innovación y del conocimiento o cualquier aspecto del desempeño de las empresas, debían apoyar directa y /o indirectamente el tejido empresarial de la región, recogiendo la idea de que un SRCTI debe consolidar una atmósfera industrial (Marshall, 1920; Morgan, 1997).

Así mismo, se eligieron como vínculos aquellos que responden directa o indirectamente a alguno de los esfuerzos de concertación llevados a cabo en el Valle del Cauca; y por último, que los enlaces debían generar corresponsabilidad entre las partes en el manejo y asignación de los recursos durante un tiempo suficiente como para que las organizaciones del SRCTI desarrollen confianza. Este ultimo principio trata de capturar la idea de que las redes contribuyen a fortalecer el capital social como un activo intangible que ayuda a disminuir costos en el proceso de innovación (Lundvall, 2002).

Basado en estos criterios se elaboró un cuestionario en el cual se le preguntaba a las organizaciones cuáles habían sido entre los años 2006 al 2009, los proyectos más importantes en materia tecnológica o de producción que habían emprendido en colaboración con otra organización de la región, del país o de cualquier parte del mundo. A renglón seguido se les pidió que señalaran el nombre de las organizaciones 
colaboradoras. También se les solicitó a las organizaciones que indicaran qué otras empresas participaban en la junta directiva u órgano de decisión. De igual forma, se les indagó si la organización participaba en alguna mesa o comité que tuviera como objetivo la coproducción, gestión y transferencia de conocimiento e innovación. Por último, se consultó a las organizaciones sobre cuáles eran las modalidades preferidas para ejecutar los proyectos de colaboración tecnológica.

\subsection{Evaluación de las características del SRCTI del Valle del Cauca}

Se construyó una matriz de adyacencia para determinar la ausencia o presencia de relaciones entre un par de organizaciones (Hanneman, 2000). La información de la matriz se introdujo en el programa Ucinet Net Draw y se calcularon parámetros sobre las características de la red. El primer paso consistió en verificar la existencia de una red entre las organizaciones del sistema y se le pidió al programa que arrojara el grafo derivado de las relaciones identificadas en la matriz de adyacencia. El segundo paso fue evaluar la conectividad y estructura del SRCTi de la región. A continuación se describe este proceso.

$1^{\circ}$. La conectividad. En este trabajo se propone usar esta propiedad como una proxy de la proximidad necesaria para el funcionamiento de los sistemas de innovación. Con la conectividad de la red se espera identificar qué tan articuladas, conectadas y cohesionadas están las organizaciones del sRCTi del Valle del Cauca. Con ese fin se calcularon tres parámetros: el de densidad de la red, el cual muestra el número de relaciones reales del total de relaciones factibles y aquí se propone como una proxy de la proximidad social e institucional; la distancia geodésica entre un par de actores, para inferir el esfuerzo que le implica a un actor establecer vínculos y relaciones con otro en el departamento, a la vez que puede ser un buen indicador de la proximidad organizacional; y finalmente, el parámetro de cohesión e influencia, con el que se pretende capturar el hecho que en un sistema de innovación, la difusión y transferencia de conocimiento entre los actores depende no sólo de las conexiones reales más eficientes, como lo muestra la distancia, sino que también depende de todas las relaciones implicadas entre los actores (Hanneman, 2000).

En este trabajo se calculó la medida del flujo máximo porque con ella se pretende capturar el proceso mediante el cual el conocimiento va de una organización a otra, en donde la conexión y transmisión de conocimiento será más fuertes entre más caminos o medios tenga una organización para llegar a otra (Casti, 1995). Por este motivo se propone la medida de flujo máximo como un parámetro de la proximidad cognitiva.

$2^{\circ}$. La estructura de la red. El primer criterio para analizar la configuración del SRCTI de la región es el de poder y centralidad. El análisis de redes sociales usa varias aproximaciones para estudiar el poder que puede tener un actor en una red. Los tres enfoques más analizados son el de grado, el de cercanía y el de intermediación (Casti, 1995; Hanneman, 2000); con estos se pueden identificar las organizaciones más poderosas a nivel local. Por su parte, los enfoques de distancias geodésicas y centralidad de flujo se dirigen a identificar a las organizaciones más poderosas, no a nivel local como con los indicadores anteriores, sino a nivel de la red global (Hanneman, 2000). Los indicadores de poder y centralidad Bonacich, por su parte, están en función de la cantidad de conexiones de una organización y las de las organizaciones de su vecindario. 
En este trabajo se utilizaron las siete medidas de poder para establecer cuáles son las organizaciones que tienen el liderazgo en el sRCTi del Valle del Cauca. Para lo cual, primero se procedió a calcular la posición que cada organización ocupó en cada una de las siete medidas de poder, luego se calculó el promedio del valor de la posición para establecer un ranking de las organizaciones más poderosas, y finalmente, con la identificación de las organizaciones líderes, se identificó el grado de jerarquía del sistema.

El segundo criterio usado para analizar la estructura de la red es la identificación de los componentes estructurales. Para describir un SRCTI es conveniente identificar aquellas organizaciones que actúan como articuladoras de éste como un todo; por ello es de particular interés buscar los agujeros, vulnerabilidades o puntos débiles en la estructura general de la red. Aquí se utiliza el enfoque denominado lambda set; el cual permite identificar las organizaciones que tienen tal nivel de conexiones que, al ser eliminadas de la red, esta se desintegraría en una estructura desconectada (Casti, 1995).

\section{Análisis y descripción del SRCTI del Valle del Cauca}

En este apartado del documento se procede a describir y analizar el SRCTI de la región a través de los parámetros de la aplicación del análisis de las redes sociales.

\subsection{Las organizaciones y sus vínculos en el SRCTI del Valle del Cauca}

Una primera aproximación para entender un sistema de innovación es identificar qué organizaciones la componen y qué vínculos establecen para producir y transferir conocimiento e innovaciones. Se encontró que en el SRCTI de la región, existen organizaciones en cada uno de los entornos que componen esta clase de sistemas. Además, se identificó que dada la misión y objetivos de las organizaciones, estas se pueden clasificar en once grupos: 1. Servicios empresariales; 2. Control, regulación de precios y tributación, 3. Financiación de la producción, 4. Brindar incentivos gubernamentales, 5 . Regulación de salarios, 6. Regulación de beneficios, 7. Generación y regulación de capital humano, 8. Innovación y generación de conocimiento, 9. Financiación de la educación y la investigación, 10. Coordinadores y articuladores de la difusión de la ciencia, la tecnología y mercado, y 11. Difusión y transferencia de conocimiento. El análisis del conjunto de actores se ilustra en la Tabla 1.

Una mirada a la Tabla 1 permite inferir que el Valle del Cauca cuenta con un tejido organizacional para emprender procesos de innovación y de apoyo a la producción. Del referenciamiento de las organizaciones del SRCTI en el entorno de relaciones interempresariales, se encuentran siete organizaciones, de las cuales cinco desempeñan funciones de regulación de beneficios y dos brindan servicios empresariales. En el entorno de actuaciones públicas en innovación, existen doce organizaciones, de las cuales seis cumplen funciones de control, regulación de precios y tributación; cuatro generan incentivos gubernamentales y dos generan financiación de la educación y la investigación. En el entorno de infraestructura de soporte a la innovación, se encuentran veintisiete organizaciones, de las cuales diez cumplen la función de difusión y transferencia de conocimiento, ocho universidades generan y regulan el capital humano, ocho tienen la función de transferir la innovación y la generación del conocimiento y por ultimo una que coordina y articula la difusión de la ciencia, la tecnología y mercados. En el entorno financiero existen doce organizaciones de las cuales nueve financian la producción, dos financian la educación y la investiga- 
Henry Caicedo Asprilla

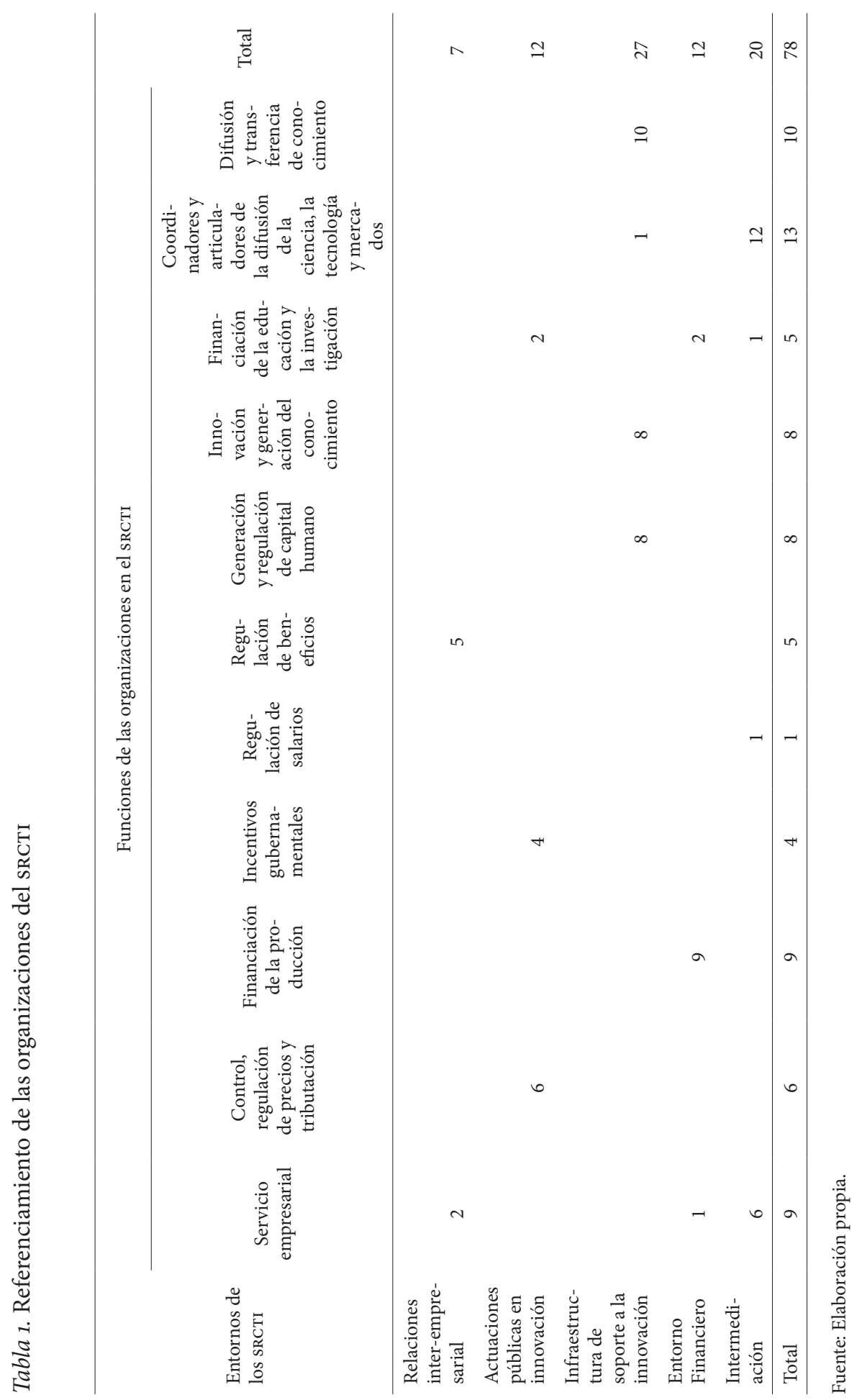


ción, y una brinda servicios empresariales. Finalmente, en el entorno de intermediación existen veinte organizaciones, de las cuales doce son coordinadoras y articuladoras de la difusión de la ciencia, tecnología y mercados; seis brindan servicios empresariales, una financia la educación y la investigación, y una regula salarios.

De una revisión profunda de la Tabla 1 se puede deducir que el Valle del Cauca ha logrado construir un tejido organizacional, lo cual constituye una fortaleza, al menos potencial, para el buen funcionamiento del sistema como un todo, en tanto que disponer de centros de desarrollo tecnológicos, de universidades, de instituciones financieras, al igual que de organizaciones de infraestructura para la innovación, permitiría en el mediano plazo crear las condiciones para que las empresas encuentren un ambiente propicio para la generación de innovaciones $y$, sin duda, de ventajas competitivas.
Esta característica es típica de sistemas de las regiones periféricas en países como Grecia, España, Portugal y el sur de Italia, donde las organizaciones se crean en respuesta a estrategias de desarrollo regional motivadas por la necesidad de impulsar una institucionalidad que apoye las empresas, fundamentalmente a las pymes (Andersson \& Karlsson, 2006; Fernández, Rojo \& Castro, 2003; Iammarino, 2005).

Sin embargo, como se ha advertido durante todo el documento, en un SRCTI no basta con disponer de las organizaciones necesarias en cada uno de los entornos, se requiere que estas establezcan vínculos a través de proyectos de cooperación para generar, producir y transferir conocimiento y crear innovaciones (Cantner et al., 2010). Al respecto, la Tabla 2 recoge un inventario de procesos de cooperación que las organizaciones consideraron como los más importantes ejecutados con otras organizaciones. Para fines de simplificar el análisis, se clasificaron

Tabla 2. Inventario de procesos de cooperación entre las organizaciones del SRCTI para apoyar a las empresas

\begin{tabular}{lcc}
\hline \multirow{2}{*}{ Vínculos de las organizaciones en el SRCTI } & \multicolumn{2}{c}{ Proyectos por funciones } \\
\cline { 2 - 3 } & Número & $\%$ del total \\
\hline Servicios empresariales & 29 & $32,2 \%$ \\
Control, regulación de precios y tributación & 7 & $7,8 \%$ \\
Financiación de la producción & 6 & $6,7 \%$ \\
Incentivos gubernamentales & 7 & $7,8 \%$ \\
Regulación de salarios & 0 & $0,0 \%$ \\
Regulación de beneficios & 1 & $1,1 \%$ \\
Generación y regulación de capital humano & 10 & $11,1 \%$ \\
Innovación y generación del conocimiento & 2 & $2,2 \%$ \\
Financiación de la educación y la investigación. & 8 & $8,9 \%$ \\
Coordinadores y articuladores de la difusión de la ciencia, la tecnología y mer- & & $13,3 \%$ \\
cados & 12 & $8,9 \%$ \\
Difusión y transferencia de conocimiento & 8 & $100,0 \%$ \\
\hline Total & 90 & \\
\hline
\end{tabular}

Fuente: Elaboración propia. 
en las necesidades del sistema que, según las organizaciones, deben resolverse para apoyar a las empresas.

Una mirada a la Tabla 2 permite deducir que las organizaciones del SRCTI celebraron noventa acuerdos de cooperación para resolver problemas a las empresas y a los factores de producción de la región en el período 2006-2009. Dada la naturaleza de la Tabla 2, de aquí se puede inferir que la principal razón por la que las organizaciones del SRCTI establecen convenios de cooperación, es para brindar servicios empresariales (32,2\% de los proyectos); la segunda motivación de vinculación entre las organizaciones es la del establecimiento de acuerdos para coordinadores y articuladores de la difusión de la ciencia, la tecnología y mercados $(13,3 \%)$. Al respecto, es de resaltar que, en su mayoría, esta clase de vínculos se asocia a la conformación de mesas de trabajo, Consejos como el de competitividad y el de bioregión, comités de diálogo y coordinación de tareas como el CARCE, y mesas técnicas como el CODECTI.

El tercer tipo de interés más recurrente por el que las organizaciones se unen es para la generación y regulación de capital humano ( $11,1 \%$ de los proyectos); le siguen en orden de mayor a menor motivación, la financiación de la educación $(8,9 \%)$ y la difusión en transferencia de conocimiento $(8,9 \%)$, los convenios para crear incentivos gubernamentales y el control, regulación de precios y tributación $(7,8 \%)$, la financiación de la producción $(6,7 \%)$, la generación del conocimiento $(2,2 \%)$; y por último, los acuerdos para la regulación de beneficios $(1,1 \%)$.

Como los datos lo indican, las organizaciones del SRCTI de la región se buscan principalmente para brindarse servicios empresariales, mientras que las razones de innovación e investigación apenas si suman el 10\% de las motivaciones de vinculación. Este hallazgo permite inferir que las vinculaciones no tienen por objeto la transferencia y/o producción de conocimiento y la innovación, de aquí que la red formada no es una red de conocimiento propiamente dicha, más bien es una red de apoyo empresarial, concentrada más en temas de asistencia técnica, capacitaciones, consultorías, a la vez que para financiar la producción, regular salarios y beneficios, entre otros problemas asociados al desempeño empresarial, mientras que los temas de innovación, investigación y generación de conocimiento todavía no motiven a las organizaciones del SRCTI para establecer procesos de cooperación.

De los resultados de la Tabla 1 y la Tabla 2 puede deducirse que el SRCTI del Valle del Cauca tiene la potencialidad de ser un sistema que, eventualmente, podría ayudar a mejorar las condiciones de innovación y gestión del conocimiento, en tanto que posee un tejido organizacional en el cual se identifican entidades que operan en función de las necesidades de las empresas de la región; en el Valle del Cauca existe una proximidad geográfica ya que el $90 \%$ de las organizaciones se concentran en Cali y en su área de influencia. No obstante esta potencialidad, actualmente el SRCTI es un sistema débil porque las organizaciones no tienen como prioridad celebrar convenios y acuerdos para generar, producir y transferir conocimiento e innovación.

La debilidad del sRCTI en el Valle del Cauca radica en que, dadas las prioridades de establecimientos de procesos de cooperación, el sistema experimenta fallas de los sistemas de innovación, asociadas a la existencia de organizaciones con funciones no adecuadas para la innovación. A su vez, el sistema experimenta la falla de generar interacciones no necesarias y no conducentes 
para la gestión del conocimiento. En estas condiciones, el sistema no puede ayudar a las empresas a facilitar las interacciones entre agentes, ni proporcionar recursos para la innovación, ni dinamizar la transferencia de tecnología y conocimiento desde y hacia el exterior de la región. Sistemas de esta clase son semejantes a los de las regiones periféricas de Europa, que se caracterizan por tener una trayectoria de dependencia tecnológica, es decir, por poseer sectores dominados por la oferta y poco innovadores, y como resultado, las organizaciones se concentran más en la generación de servicios (Fernández et al., 2003; Vence \& González, 2002).

\subsection{Análisis de la conectividad del SRCTI del Valle del Cauca}

En este apartado se describen los hallazgos en la estructura del SRCTI una vez aplicada la metodología de redes. El análisis se inicia con la descripción de la red construida a partir de las relaciones identificadas en función de los noventa procesos de cooperación. La red se muestra en el Gráfico 1.

El Gráfico 1 muestra la red de relaciones entre las organizaciones clasificadas según el entorno al que pertenecen y el papel o función que tienen en el sRCTI. Así, las del entorno de relaciones inter-empresariales se representan con círculos, las de infraestructuras de innovación con triángulos, etc. Por su parte, el rol o función de las organizaciones se distingue por escala de grises. Las organizaciones relacionadas con doble flecha son aquellas que han constituido proyectos de cooperación con otra, donde ambas han colocado recursos, y aquellas enlazadas con flecha en una sola dirección significa que una organización coloca recursos para el desarrollo de proyectos con otra o que participa en sus órganos de decisión.

Un ojo entrenado en el análisis de grafos podrá detectar que son más las relaciones de doble vía entre las organizaciones que las unidireccionales. Por ejemplo, el Gráfico 1 evidencia que la Red del SRCTI de la región se asemeja a un mapa de carreteras, lo cual implica que es una red descentralizada, es decir, que el sistema no es monocéntrico sino policéntrico. Ahora bien, dado el tipo de proyectos de colaboración que se emprenden en la región, en esta clase de redes se suele presentar como problema la duplicación de esfuerzos, con el consiguiente desperdicio de recursos y el bajo impacto en el desarrollo tecnológico.

Para analizar más profundamente la conectividad del SRCTI y explicar la forma del grafo, basta con evaluar las propiedades básicas de la red. En la Tabla 3 se describen los parámetros de las propiedades del sRCTI.

La densidad promedio de la matriz de adyacencia es 0,107 , lo que quiere decir que sólo el $10,7 \%$ de las conexiones posibles están presentes en la red estudiada. Adicionalmente, a partir de los 1 y o de la matriz de adyacencia, que representan la presencia o no de conexión, se puede calcular una desviación estándar de 0,309, es decir, existe relativamente una pequeña variación en las relaciones, lo que está asociado con la baja densidad que presenta la red. De los resultados de la Tabla 3 se infiere que el SRCTI de la región es un sistema con muy baja capacidad de relacionamiento, como lo evidencia el hecho que para el período de tres años, las organizaciones sólo reportaron noventa proyectos de cooperación; esta es una evidencia más de la poca tendencia a la cooperación, lo cual significa que el sistema tiende más a la desconexión que a la conectividad entre los agentes. La fragilidad del sistema se evidencia en el reconocimiento que existe una débil proximidad institucional y social, porque aunque estaba vigente la Ley 29 (Congreso de la Republica de Colombia, 1990) y sus decretos reglamentarios, estos 
Gráfico 1. Grafo de la red del sRcti del Valle del Cauca

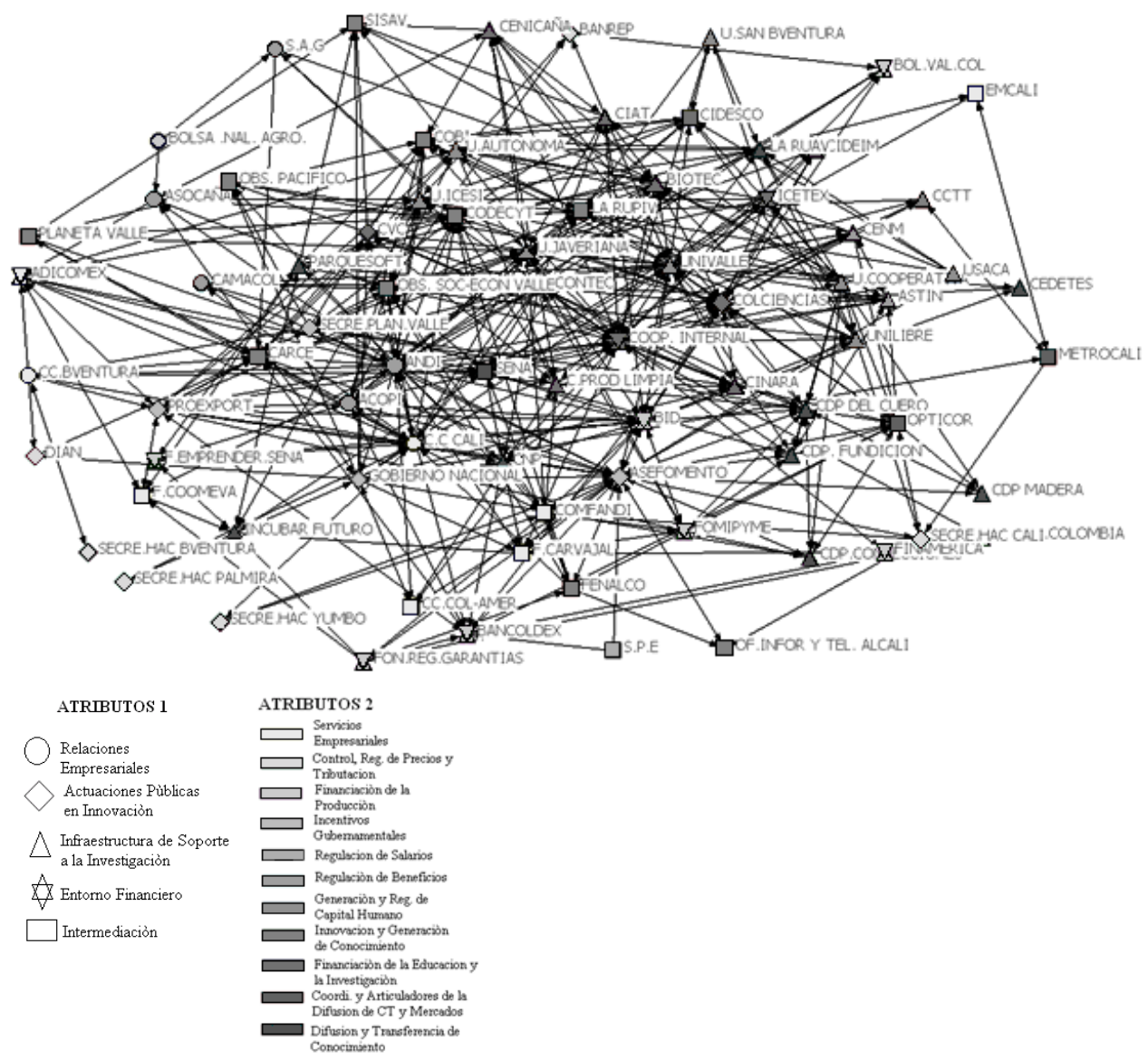

Fuente: Elaboración propia.

Tabla 3. Indicadores básicos para el análisis de la red

\begin{tabular}{lc}
\hline Indicador & Valor \\
\hline Conexiones existentes & 638 \\
Conexiones posibles & 5.852 \\
Densidad (promedio matriz) & 0,107 \\
Desviación estándar & 0,309 \\
Distancia media (entre pares alcanzables) & 2,336 \\
Cohesión basada en distancia (compactación) & 0,456 \\
Fragmentación ponderada por distancia (amplitud) & 0,544 \\
\hline
\end{tabular}

Fuente: Elaboración propia. 
no se constituyen en incentivos para generar acuerdos de cooperación, lo que no permite incrementar el capital social.

Si bien la densidad proporciona información sobre la fortaleza de un sistema, interesa saber qué tanto esfuerzo le implica a una organización establecer vínculos de cooperación con otra. En este sentido, el análisis de redes proporciona información sobre este aspecto a través del cálculo de la distancia geodésica, como se muestra en la Tabla 4, la cual presenta el promedio de pasos que debe dar una organización para establecer vínculos con otra.

Como se evidenció en la Tabla 3, en promedio, la distancia geodésica entre las instituciones analizadas es de 2,336, el nivel de cohesión debida a esta distancia es de un $45,6 \%$ y el de fragmentación de $54,4 \%$. Adicionalmente, la Tabla 4 percibe que la distancia más larga entre un actor y otro es de 5 relaciones, la distancia más frecuente es de una longitud de 2 (con el 49,7\%), le sigue una distancia de longitud de 3 , luego de 1 , seguida por $4 \mathrm{y}$ finalmente, la menos frecuente es la distancia de una longitud de 5 (con el o,2\%).

Los resultados de la Tabla 4 corroboran que el SRCTI está en fase de consolidación. Sobre esto, la debilidad del sistema asociada a estos parámetros radica en que la distancia necesaria entre un par de organizaciones en un SRCTI fuerte para garantizar el aprendi-

Tabla 4. Frecuencias de distancias geodésicas

\begin{tabular}{lcc}
\hline Longitud & Frecuencia & Proporción \\
\hline 1 & 641.000 & 0,114 \\
2 & 2.797 .000 & 0,497 \\
3 & 1.859 .000 & 0,330 \\
4 & 318.000 & 0,057 \\
5 & 12.000 & 0,002 \\
\hline
\end{tabular}

Fuente: Elaboración propia. zaje, tiende a la unidad (Lundvall, 2002), y la máxima es de cinco (Granovetter, 1985), mientras que en la región es de 2,3; por lo que no se presenta con frecuencia la relación cara a cara necesaria para la transmisión de conocimiento (Lundvall, 2002).

Otro rasgo que muestra que el SRCTI del Valle del Cauca es frágil, es el hecho que la red experimenta un nivel de fragmentación de $54,0 \%$, lo cual muestra que es un sistema que impone esfuerzos a las organizaciones adicionales a los necesarios para ejecutar procesos de cooperación, aún en los más elementales, como los asociados a los servicios empresariales. Este resultado corrobora que aunque en la región existe proximidad geográfica, es relativa la proximidad organizacional porque, aunque existen organizaciones, la probabilidad de ejecutar con éxito un proceso de innovación disminuye por la distancia geodésica entre estas.

Un tercer aspecto, necesario para evaluar un SRCTI, es la identificación del grado de accesibilidad existente entre las organizaciones a través de la medida del flujo máximo (Hanneman, 2000). Los resultados asociados a esta medida se muestran en el Gráfico 2.

En el caso de la red estudiada, como se muestra en el Gráfico 2, la gran mayoría de los actores encuentran hasta cinco caminos para llegar a otro actor y son muy pocos los que encuentran más de diez formas diferentes para alcanzar a otro actor. La institución que cuenta con el mayor flujo máximo es la ANDI con hasta veintisiete formas diferentes para llegar hasta Cooperación Internacional, le siguen con veintiséis rutas de nuevo ANDI hasta Univalle y Cooperación Internacional hasta Univalle también con veintiséis rutas.

Como dato particular, se encontró que CARCe, la RUAV y el Observatorio del Pacífico cuentan con un flujo máximo de cero, es decir, no encuentran ningún camino de 
Gráfico 2. Flujo máximo de las conexiones de la red institucional

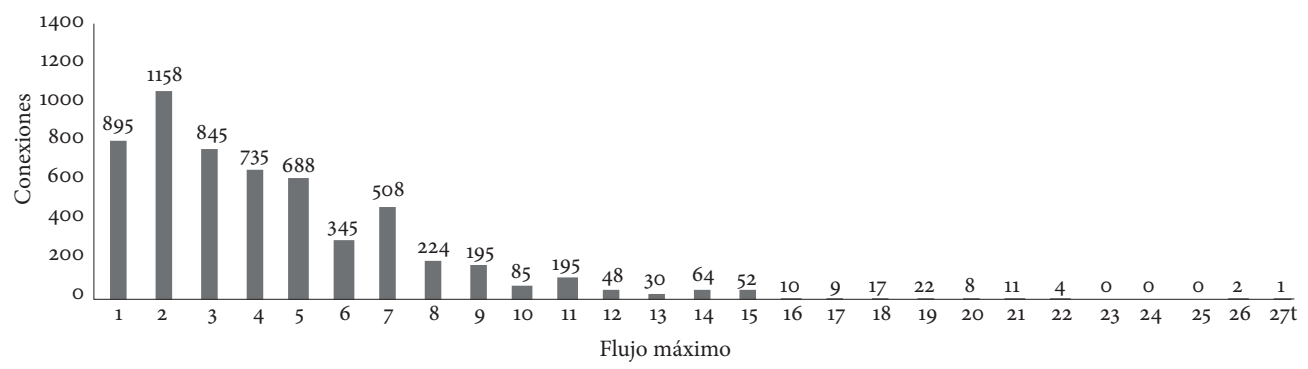

Fuente: Elaboración propia.

trasmisión a las demás organizaciones. Por su parte, la DIAN, la Secretaria de Hacienda de Buenaventura y la Universidad Autónoma, cuentan con sólo una organización, que por lo general, le sirve de trasmisora. Por otra parte ADICOMEx, la Cámara ColomboAmericana, el Centro de Producción Limpia, el CCTT, CDP Confecciones, el Fondo Emprender sENA, el Fondo de Garantía y Planeta Valle, encuentran hasta cinco organizaciones en su vecindario para formar vínculos organizacionales.

De aquí se puede inferir que la accesibilidad como proxy de la proximidad cognitiva, indica que el sistema tiene un bajo índice en este aspecto porque, dado el número de organizaciones, el que cinco sea el promedio de caminos o formas para conectarse entre un par de organizaciones, indica que el sistema tiene un flujo máximo de información bajo y de poca accesibilidad.

Tomando como parámetros de análisis la densidad, la distancia geodésica y la accesibilidad, se puede afirmar que el sRCTI de la región está en fase de consolidación y es débil. Esto se debe a que el sRcti en el Valle del Cauca experimenta una baja propensión al establecimiento de vínculos, a que las organizaciones deben hacer grandes esfuerzos para establecer procesos de cooperación con otras, a que el sistema es fragmentado y a que la accesibilidad es relativamente baja para la gran mayoría de organizaciones.
Igualmente, este sistema experimenta problemas de proximidad institucional, social, organizacional y cognitiva, lo que parece ser un problema de las regiones colombianas. $\mathrm{Al}$ respecto, Amar \& Díaz (2006) encontraron evidencia en el departamento del Atlántico sobre el SRCTI como un sistema desarticulado, desequilibrado y débil. Este tipo de sistemas tendrán dificultades para reducir la incertidumbre que entraña el proceso de innovación en tanto que no hay una propensión a acompañar a las empresas o estas no buscan cooperación para emprender innovaciones, de aquí que el gasto en $\mathrm{I}+\mathrm{D}+\mathrm{i}$ sea bajo en las empresas y las organizaciones de apoyo (Andersson \& Karlsson, 2006).

\subsection{Análisis de la estructura del SRCTI del Valle del Cauca}

Para avanzar un paso más en la comprensión del sRCTi del Valle del Cauca, se analizan los hallazgos obtenidos en dos aspectos de la red: en las medidas de poder y centralidad, $y$ en el análisis de los componentes estructurales. Para el análisis y posterior intervención de un SRCTI, es relevante identificar las organizaciones que ostentan mayor poder, tanto a nivel local como global (Gertler \& Levitte, 2003). Una aproximación a este fenómeno en el sRCTi del Valle del Cauca se ilustra en la Tabla 5, este muestra una síntesis de las diez instituciones más poderosas del sistema. 
Como se muestra en la Tabla 5 , se tiene que el BID es la organización con mayor poder, seguida de Univalle y Asesoría de Fomento Económico y Competitividad. En general, se encuentran dos financiadoras de la educación y la investigación, dos universidades, una incentivadora gubernamental, una prestadora de servicios interempresariales, una financiadora de la producción, una reguladora de beneficios, una generadora de conocimiento y una difusora de conocimiento como las instituciones que cuentan con mayor poder.

Para complementar este análisis, el Gráfico 3 muestra las organizaciones que si se eliminan del SRCTI, generan un efecto perturbador a tal punto que distorsionan y desconectan al sistema como un todo.
De acuerdo a los resultados de este enfoque y como se puede constatar en la Gráfico 3, se encuentra que los vínculos 36 y 77, Cooperación Internacional y Univalle, respectivamente, son los más importantes del grafo porque tienen el mayor valor de lambda set equivalente a 32, lo cual significa que son las organizaciones que al eliminarse, perturbarían enormemente el grafo dada la cantidad de vículos que poseen.

Los resultados de la Tabla 5 y el Gráfico 3 tienen una doble interpretación: en primer lugar, el tipo de entidades que lideran el SRCTI (universidades, instituciones públicas y organizaciones para la cooperación internacional) son un rasgo positivo porque permitirán que éste avance a partir de procesos de concertación donde estas sean entidades dinamizadoras. Esta es una lectura optimista

Gráfico 3. Jerarquía de posiciones de conjuntos Lambda

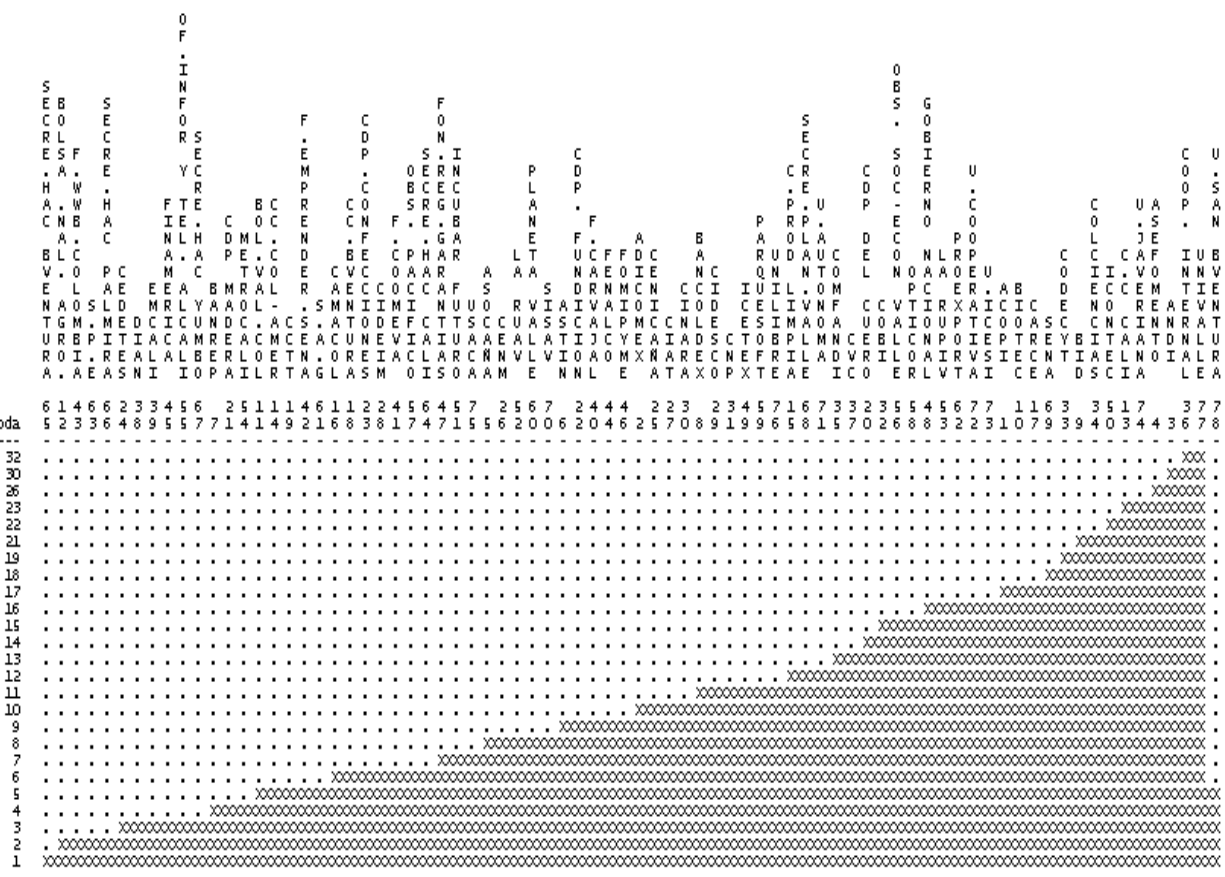

Fuente: Elaboración propia. 


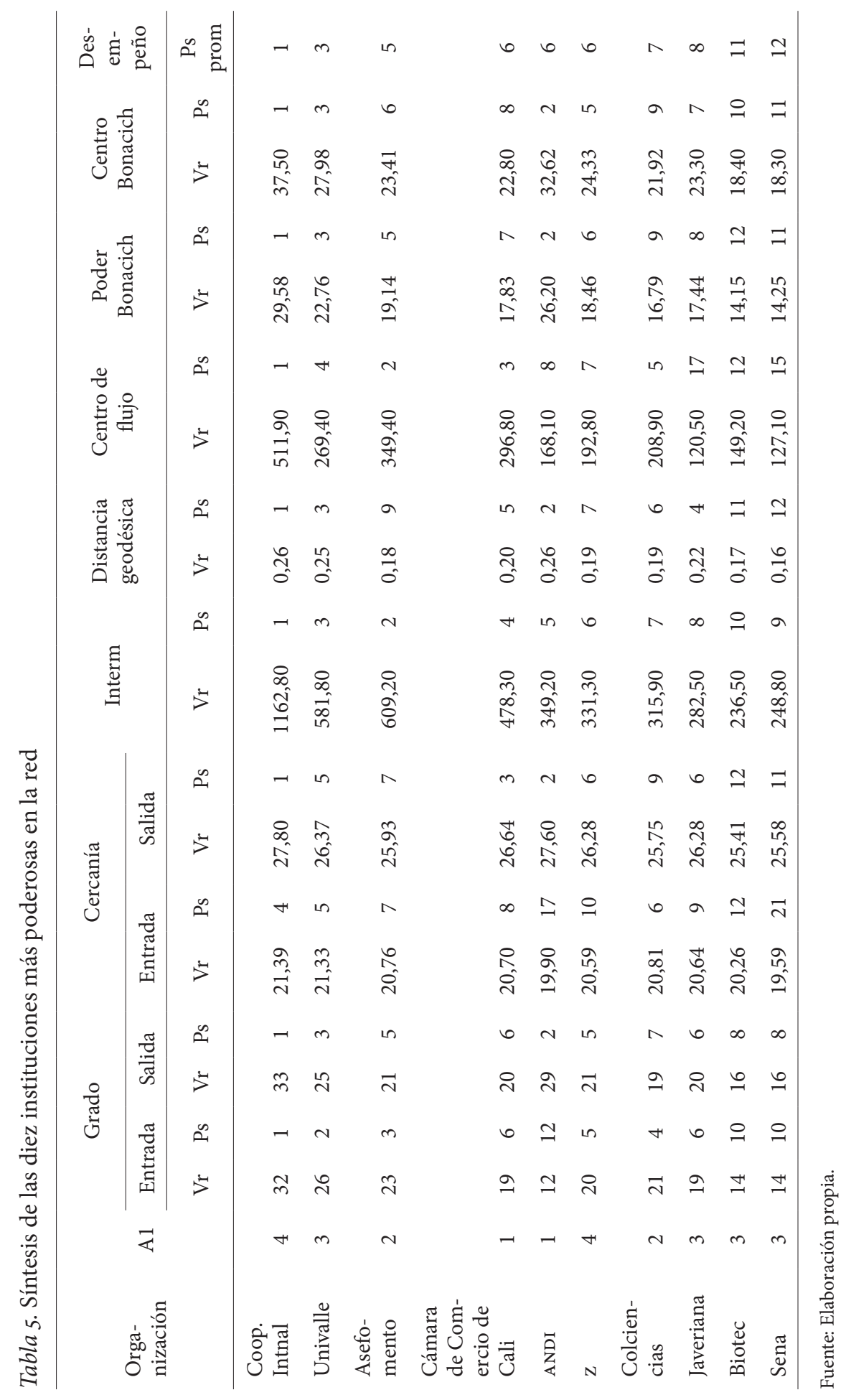


en tanto que se evidencian potencialidades a futuro en el sistema.

En segundo lugar, desde la lógica funcional del sistema, el que las entidades líderes sean en su mayoría públicas y de cooperación internacional, hacen del SRCTI de la región un sistema altamente vulnerable pues dependen de aspectos exógenos, como en el caso del BID si disminuyera su influencia, en el caso de Univalle si se presentara una crisis institucional o en Fomento Económico, que la administración cambiara de intereses. La presencia de la ANDI y de algunas entidades privadas no son garantía de funcionamiento tampoco, más bien son un reflejo de la importancia que tienen los gremios como reguladores de beneficios y la Cámara de Comercio como el principal generador de servicios empresariales en el SRCTI de la región.

Este tipo de sistemas experimenta fallas de mercados como monopolios ostentados por los gremios, asimetría de información asociada al diferencial de capacidades de absorción de conocimiento entre las universidades y empresas; al igual que existe diferencial en la especificidad de los activos tangibles e intangibles necesarios para la innovación (Iammarino, 2005). En estas condiciones el mercado no es el regulador y asignador de los recursos para la innovación, esto es, que la iniciativa privada no es determinante en la generación de conocimiento, lo cual va en contra vía de la necesidad que impone la nueva economía, en la que cada vez más los gastos en $\mathrm{I}+\mathrm{D}+\mathrm{i}$ provengan de las empresas y no de los entes públicos o de cooperación (Casas, 2001).

\section{Conclusiones}

La principal reflexión que puede derivarse del presente trabajo es que en las regiones como la del Valle del Cauca, región relevante de un país en vía de desarrollo como
Colombia, los llamados SRCTI son más una aspiración de orden político que una realidad fáctica. En este orden de ideas, el Valle del Cauca es un caso en el que se dispone de las instituciones y las organizaciones pero no se cuenta con las redes necesarias para que el sistema se comporte como un todo integrado. De aquí que en la región apenas si se está consolidando una infraestructura que más parece orientada a la gestión empresarial que a la innovación.

También se puede inferir que el sRCTI de la región, si se puede seguir llamando así, tiene como principal fortaleza que cuenta con un tejido institucional y organizacional; y con entidades que eventualmente podrían ayudar a acelerar la consolidación del sistema. Sin embargo, debe tenerse en cuenta que para ello deberá superar al menos tres problemas: la baja conectividad entre las organizaciones, la alta vulnerabilidad al tener instituciones con un gran efecto de arrastre y, por último, cambiar la trayectoria de desarrollo, pues se necesita pasar de un sistema que presta servicios empresariales de bajo contenido tecnológico a una trayectoria orientada hacia la innovación.

Una importancia significativa de esta investigación es haber logrado evaluar al SRCTI usando la metodología del análisis de redes sociales; de esta manera, se logró avanzar hasta mostrar que un SRCTI es una red y, que por tanto, la metodología puede ayudar a explicar las principales características de este; lo que permitió identificar los puntos débiles y fuertes del sRCTI. Además, permitió dar cuenta de lo más importante de un sistema, como lo es el grado de articulación y, en segundo término, de algunos rasgos de su estructura.

No obstante las virtudes de usar el análisis de las redes sociales, existen elementos limitantes en los que se debe enfatizar en posteriores trabajos similares, el más rele- 
vante es que una red es una especie de foto instantánea del estado del SRCTI; así que el surgimiento de una nueva organización o de una nueva institución formal como una ley, hará que la red cambie y con ello las inferencias y deducciones que puedan derivarse.

De igual manera, la aplicación del análisis de las redes sociales, como estudio cualitativo, depende mucho de la información de los entrevistados, así que siempre es posible que algunos vínculos puedan omitirse o ponderarse, lo cual tiene un efecto en la identificación y cálculos de las propiedades de la red; por ello se recomienda que los resultados sean evaluados con cautela y evaluar buscando la generalidad más que aspectos puramente particulares de una organización.

De este trabajo quedan dos interrogantes que pueden convertirse en futuras líneas de investigación: ¿Qué relación existe entre los parámetros de las redes como densidad, distancia geodésica y accesibilidad con los resultados e indicadores de eficiencia de los SRCTI como generación de patentes, nuevos productos, formación de recursos humanos, entre otros? ¿Qué ocurre con la institucionalidad de países como Colombia, que por una parte incentivan la creación de las organizaciones para apoyar a la innovación, pero por otra no incentivan a la articulación entre ellas?

\section{Referencias bibliográficas}

Amar, P. \& Díaz, J. (2006). Modelo de relación Estado-mercado para el fomento de la innovación. Investigación y Desarrollo, 14(1), 3-25.

Andersson, M. \& Karlsson, C. (2004). The Role of Accessibility for Regional Innovation Systems. En C. Karlsson, P. Flensburg, \& S.-A. Hörte (Eds.), Knowledge Spillovers and Knowledge Management, Edward Elgar, Cheltenham (in press).

Andersson, M. \& Karlsson, C. (2006). Regional Innovation Systems in Small and Medium-Sized Regions. En B. Johanson, C. Karlsson, \& R. Stough (Eds.), The Emerging Digital Economy, entrepreneurship, Clusters \& Policy (pp. 55-81). Berlin: Springer-Verlag.

Asheim, B. \& Coenen, L. (2004, junio). The role of regional innovation systems in a globalizing economy: Comparing knowledge bases and institutional frameworks of Nordic clusters. Documento presentado en DRUID Summer Conference, Rebild, Dinamarca.

Asheim, B. \& Isaksen, A. (2001). Los sistemas regionales de innovación, las pymes y la política de innovación. En M. Olazarán \& M. Gómez (Eds.), Sistemas regionales de innovación (pp. 93-114). Bilbao, España: Universidad del País Vasco.

Asheim, B. \& Isaksen, A. (2002). Regional Innovation Systems: The Integration of Local 'Sticky' and Global 'Ubiquitous' Knowledge. The Journal of Technology Transfer, 27(1), 77-86.

Becattini, G. (1990). The Marshallian Industrial District as a Socio-Economic Nation. En F. Pyke, G. Becattini \& W. Sengenberger (Eds.), Industrial Districts and Inter-Firm Co-operation in Italy (pp. 37-51). Ginebra, Suiza: ILO.

Bell, M. \& Pavitt, K. (1993). Technological Accumulation and Industrial Growth: Contrasts between Developed and Developing Countries. Industrial and Corporate Change, 2(2), 185-203.

Boschma, R. (2005). Proximity and innovation: a critical assessment. Regional Studies, 39(1), 61-75.

Buesa, M., Martínez, M., Heijs, J. \& Baumert, T. (2002). Los sistemas regionales de innovación en España. Una tipología 
basada en indicadores económicos e institucionales. Revista Economía Industrial, 347, 15-32.

Cantner, U., Meder, A. \& Ter Wal, A.J. (2010). Innovator networks and regional knowledge base. Technovation, 3o(9-10), 496-507.

Casas, R. (2001). Espacios emergentes de conocimiento en las regiones: Hacia una taxonomía. En La formación de redes de conocimiento: Una perspectiva regional desde México (pp. 35-73). México: Anthropos.

Cassiolato, J.E. (2002, marzo). Principais Características a Investigar em uma pesquisa de Inovação. Estudio Metodológico. Documento presentado en La Encuesta de Innovación Tecnológica en las Empresas, Rio de Janeiro, Brasil.

Casti, J. (1995). Complexification. New York, NY: Harper Perennial.

Carlsson, B. (2006). Internationalization of innovation systems: A survey of the literature. Research policy, 35(1), 56-67.

Consejo Nacional de Política Económica y Social-CONPES. (2000). CONPES 3080: Política Nacional de Ciencia y Tecnología. Recuperado de http://ocyt.org.co/html/ archivosProyectos/conpes\%2ocyt.pdf

Cooke, P. (2001). Regional Innovation Systems, Clusters, and the Knowledge Economy. Industrial and Corporate Change, 10(4), 945-974.

Cooke, P. (2004). Regional Innovation Systems - an evolutionary approach. En P. Cooke, M. Heidenreich \& H.J. Braczyk (Eds.), Regional innovation systems: the role of governance in a globalized world (pp. 1-20). Londres: Routledge.

Cooke, P., Gómez, M. \& Etxebarria, G. (1997). Regional innovation systems: Institutional and organizational dimensions. Research Policy, 26(4-5), 475-491.
DeBresson, C. \& Amesse, F. (1991). Networks of innovators: A review and introduction to the issue. Research Policy, 20(5), 262-279.

Dosi, G. (1999). Some Notes in National Systems of Innovation and Production and their Implications for Economic Analysis. En D. Archibugi, J. Howells \& J. Michie (Eds.), Innovation Policy in a Global Economy (pp. 35-48). Cambridge: Cambridge University Press.

Edquist, C. (2001, junio). The systems of innovation approach and innovation policy: an account of the state of the art. Documento presentado en DRUID Conference, Aalborg, Dinamarca.

Edquist, C. \& Johnson, B. (1997). Institutions and Organizations in Systems of Innovation. En C. Edquist (Eds.), Systems of innovation: technologies, institutions, and organizations (pp. 41-63). Londres: Routledge.

Fernández, I. \& Castro, E. (1995). La nueva política de articulación del Sistema de Innovación en España. Documento presentado en vi Seminario Latinoamericano de Gestión Tecnológica, ALTEC, Concepción, Chile.

Fernández, I., Rojo, J. \& Castro, E. (2003). Enfoques de políticas regionales de innovación en la Unión Europea. Documento de trabajo de la Academia Europea de Ciencias y Artes. Madrid: Academia Europa de Ciencias y Artes.

Florida, R. (1995). Toward the learning region. Futures, 27(5), 527-536.

Freeman, C. (1987). The economics of industrial innovation. Londres: Routledge.

Freeman, C. (1991). Networks of Innovations: A Synthesis. Research Policy, 20(5), 499-514.

Gertler, M. \& Levitte, Y. (2003). Local nodes in global networks: The geography of knowledge flows in biotechnology 
innovation. Documento presentado en Conferencia DRUID Summer, Elsinore, Dinamarca.

Granovetter, M. (1985). Economic action and social structure: the problem of embeddedness. American Journal of Sociology, 91(3), 481-510.

Hanneman, R. (2000). Introducción a los métodos de análisis de redes sociales. Disponible en http://www.redes-sociales.net/

Hekkert, M.P., Suurs, R.A.A., Negro, S.O., Smits, R.E.H.M. \& Kuhlmann, S. (2007). Functions of innovation systems: a new approach for analyzing technological change. Technological Forecasting and Social Change, 74(2007), 413-432.

Iammarino, S. (2005). An evolutionary integrated view of regional systems of innovation. Concepts, measures and historical perspectives. European Planning Studies, 13(4), 495-517.

Isaksen, A. (2001). Building Regional Innovation Systems: Is endogenous industrial development possible in the global economy? Canadian Journal of Regional Science, 24(1), 101-120.

Jaffe, A.B., Trajtenberg, M. \& Henderson, R. (1993). Geographic localization of knowledge spillovers as evidenced by patent citations. The Quarterly Journal of Economics, 108(3), 577-598.

Koschatzky, K. (2002). Fundamentos de la economía de redes. Especial enfoque a la innovación. Revista Economía Industrial, $346,15-26$.

Ley 29, por la cual se dictan disposiciones para el fomento de la investigación científica y el desarrollo tecnológico y se otorgan facultades extraordinarias, Congreso de la Republica de Colombia. (1990). Disponible en http://www.alcaldiabogota. gov.co/sisjur/normas/Norma1.jsp?i=254

Lorentzen, A. (2009). Las redes de conocimiento en el espacio: Reflexiones de una geógrafa sobre la literatura de los sesteas regionales de innovación. Ekonomiaz, 70, 2-12.

Lundvall, B.A. (1992). National Systems of Innovation: Towards a theory of innovation and interactive learning. Londres: Pinter.

Lundvall, B.A. (1999). La base del conocimiento y su producción. Ekonomiaz, 45, 14-37.

Lundvall, B.A. (2002). Estados-nación, capital social y desarrollo económico. Un enfoque sistémico de la creación de conocimiento y el aprendizaje en la economía global. Revista de Economía Mundial, 7, 69-90.

Lundvall, B.A. (2007). National innovation system - analytical concept and development tool. Industry and innovation, 14(1), 95-119.

Lundvall, B.A. \& Johnson, B. (1994). Sistemas nacionales de innovación y aprendizaje institucional. Comercio Exterior, 44(8), 695-704.

Lundvall, B.A \& Lorenz, A. (2009). El Enfoque Dur y de Variedades Relacionadas: Innovación y desarrollo de competencias en la economía del aprendizaje. Implicaciones para las políticas de innovación. En M.D. Parrilli (Ed.), Innovación y aprendizaje: lecciones para el diseño de políticas (pp. 23-50). Zamudio: Innobasque.

Marshall, A. (1920). Principios de Economía ( $3^{\text {a }}$ ed.). Madrid: Aguilar.

Metcalfe, J. (1998). Evolutionary Economics and Creative Destruction. Londres: Routledge.

Molina, J.L. (2000). El análisis de redes sociales. Aplicaciones al estudio de la cultura en las organizaciones. Tesis doctoral no publicada, Universidad Autónoma de Barcelona, Barcelona, España. 
Morgan, K. (1997). The Learning Region: Institutions, Innovation and Regional Renewal. Regional Studies, 31(5), 491-504. Morgan, K. (2004). The exaggerated death of geography: learning, proximity and territorial innovation systems. Journal of Economic Geography, 4(1), 3-21.

Navarro, M. \& Gibaja, J.J. (2009). Las tipologías en los sistemas regionales de innovación. El caso de España. Ekonomiaz, 70, 240-281.

Porter, M.E. (1997). Estrategia competitiva: técnicas para el análisis de los sectores industriales y de la competencia. México DF: Continental.

Powell, W.W. (1990). Neither market nor hierarchy: Network forms of organization. Research in organizational behavior, 12, 295-336.

Romer, P. (1986). Increasing Returns and Long-Run Growth. The Journal of Political Economy, 94(5), 1002-1037.

Sánchez, M.P. \& Elena, S. (2006). Intellectual Capital in Universities. Improving Transparency and Internal Management. Journal of Intellectual Capital, 7(4), 529-549.

Schumpeter, J.A. (1996). Capitalismo, socialismo y democracia. Barcelona: Folio.

Utterback, J. (2001). Dinámica de la innovación tecnológica. Madrid: Fundación Cotec para la innovación tecnológica.

Velasco, E. (2002). El papel de las organizaciones de apoyo a la innovación en los sistemas de innovación regional: Reflexiones sobre su diseño y funcionamiento. Ekonomiaz, 50, 2-15.

Vence, X. \& González, M. (2002). Los servicios y la innovación. La nueva frontera regional en Europa. Economía Industrial, 347, 41-66.

Yoguel, G., Borello, J. \& Erbes, A. (2005). Conglomerados y desarrollo de sistemas locales de innovación. Observatorio de Ciencia y Tecnología de la SECYT. Dispo- nible en http://biblioteca.municipios.unq. edu.ar/modules/mislibros/archivos/23Congloprod.pdf 